\section{Sample return reveals that the asteroid Ryugu is a carbon-rich primitive body}

\section{Samples returned from the carbonaceous (C-type) asteroid 162173 Ryugu by the Hayabusa 2 mission were preliminarily analysed in a non-destructive manner. Their dark spectral features, small densities and absence of a high-temperature component imply that they are most similar to primitive CI group chondrites, but show some differences to known planetary materials.}

This is a summary of:

Yada, T. et al. Preliminary analysis of the Hayabusa2 samples returned from C-type asteroid Ryugu. Nat. Astron. https://doi. org/10.1038/s41550-021-01550-6 (2021).

Published online:

6 January 2022

\section{The mission}

Life and oceans on Earth today are thought to have originated from organic materials and water contained in early celestial bodies. Carbonaceous chondrites are among the most primitive meteorites and a possible source of organic materials and water on proto-Earth ${ }^{1}$. Their parent bodies have long been thought of as C-type asteroids based on their dark spectral features and infrared absorption band at $2.7 \mu \mathrm{m}$, both of which are typical of hydrous minerals ${ }^{2}$. The most straightforward way to confirm this hypothesis is to send a spacecraft to C-type asteroids to collect samples and return them to Earth for analysis. Accordingly, the Japan Aerospace Exploration Agency (JAXA) sent the asteroid explorer Hayabusa2 to the near-Earth C-type asteroid 162173 Ryugu. The spacecraft was launched in $2014^{3}$ and arrived at the asteroid in 2018, where it characterized it by remote sensing before the surface landers successfully collected samples at two different sites by the 'touch and go' method in 2019, returning to Earth in 2020. Our mission was to characterize these bulk samples without destroying them and compare our findings with those obtained from the platform of Hayabusa 2 and those obtained in laboratory experiments.

\section{The observation}

Returned samples were carefully curated in the Extraterrestrial Sample Curation Center in Sagamihara, JAXA, under inorganically and organically non-contaminated conditions inside clean chambers, and in a non-destructive manner. These samples were extracted from their container in a vacuum and preliminarily analysed under conditions of purified nitrogen, using a balance, an optical microscope, Fourier transform infrared spectroscopy, a near-infrared hyperspectral microscope (called MicrOmega), and multi-band visible microscope. These tools enabled us to describe the particle size distribution, density, porosity, and spectral and textural properties of both the bulk samples and of individual particles within it.

At $5.4 \mathrm{~g}$, the total weight of recovered samples is much higher than the minimum weight of $0.1 \mathrm{~g}$ that was aimed for during collection. The samples consist of thousands of particles, ranging in size from several $\mathrm{mm}$ down to sub $\mathrm{mm}$ (Fig. 1). Their bulk visible and infrared spectral profile shows low reflectance $(\sim 0.02)$ with a sharp absorption feature in $2.72 \mu \mathrm{m}$, indicating the presence of hydroxyl groups within phyllosilicates, major components of matrices of hydrous carbonaceous chondrites ${ }^{4}$.
No high-temperature component like chondrules or $\mathrm{Ca}-\mathrm{Al}$-rich inclusions $>0.05 \mathrm{~mm}$ in diameter were observed on the surface of the samples, which matches the characteristics of $\mathrm{CI}$ group chondrites ${ }^{4}$. The average bulk densities of the samples, calculated based on estimated volumes from micrographs, is $1,282 \pm 231 \mathrm{~kg} \mathrm{~m}^{-3}$, which is smaller than that of any known meteorites ${ }^{5}$.

\section{The interpretation}

The samples from Ryugu show dark reflectance ( 0.02), which is consistent with observations that were made using remote optical imaging and remote near-infrared spectroscopy, and with the darkest materials ever explored. Furthermore, the sharp narrow absorption at $2.72 \mu \mathrm{m}$ is also consistent with that of remote near-infrared spectroscopy. The fact that these samples have a smaller bulk density than any other known meteorites indicates that they have a higher porosity, consistent with the low average density of the asteroid and with the lower thermal inertia derived by thermal imaging. These results suggest that the Ryugu samples are representative of the whole asteroid.

Importantly, these samples are the first intact regolith samples that have been returned to Earth from C-type asteroids, rather than from meteorites, as fragile carbonaceous planetary materials in interplanetary space are usually mechanically destroyed, thermally altered, and contaminated by terrestrial materials during and/or after atmospheric entry to the Earth.

Our preliminary results using non-destructive analytical methods are informative for the initial description of the samples, which now need to undergo more detailed analyses, including detailed comparison of the samples with known planetary materials. If these samples returned from Ryugu are brand-new planetary samples, their detailed analyses could provide new insight into the origin of life and the oceans on proto-Earth, as well as into the early evolution of our Solar System. More fruitful scientific results will be presented by not only further detailed analyses of the Ryugu samples, but also by the analysis of samples returned from other primitive bodies by the current OSIRIS-REx (Origins, Spectral Interpretation, Resource Identification, Security, Regolith Explorer) mission and of samples returned from future missions, such as the MMX (Martian Moons eXploration) mission.

Toru Yada and Tatsuaki Okada, Institute of Space and Astronautical Science, Japan Aerospace Exploration Agency, Sagamihara, Japan. 


\section{EXPERT OPINION}

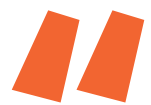

This paper is a long-awaited report on the very first nondestructive analyses of the first samples of a carbonaceous asteroid surface robotically returned to Earth. It is exciting to see these early results, and reassuring to see how well these close-up studies largely confirm the remote observations from the spacecraft platform." Beth Ellen Clark, Ithaca College, Ithaca, NY, USA.

\section{FIGURE}

a

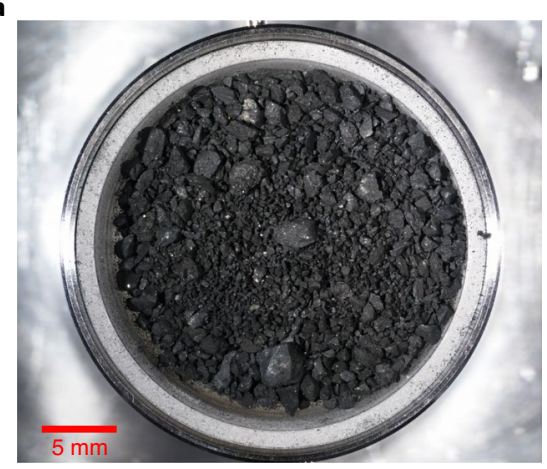

b

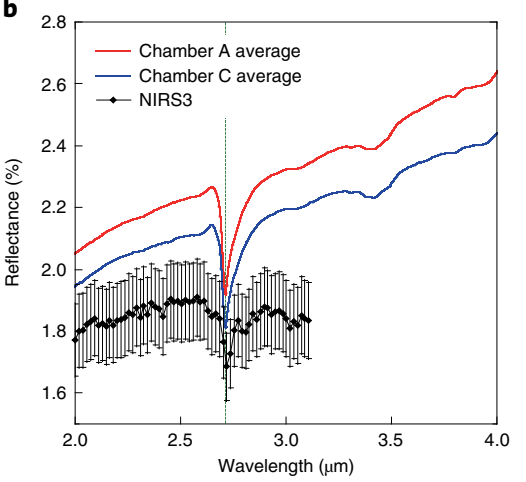

Fig. 1| Analysis of samples returned from Ryugu. a, An optical microscopy image of samples in a sapphire dish shows that they are composed of black particles that are several mm to sub-mm in size. No clear component, such as chondrule and/or Ca-Al-rich inclusions, is observed.b, The bulk visible and infrared spectrum of samples shows low reflectance ( 0.02) with sharp absorption $(2.72 \mu \mathrm{m})$, which corresponds to the presence of hydroxyl groups. The graph shows how the infrared reflectance spectra of bulk samples confirm the $2.72 \mu \mathrm{m}$ absorption feature observed with remote-sensing data for Ryugu taken by near-infrared spectrometer (NIRS3). (C) 2021, Yada, T. et al., CC BY 4.0.

REFERENCES

1. Hartman, H. et al. Carbonaceous chondrites and the origin of life. Orig. Life Evol. Biosph. 23, 221-227 (1993).

This paper reports possible sources of life from organics in carbonaceous chondrites.

2. Rivkin, A. S. et al. in Asteroids IV (eds Michel, P. et al.) 65-87 (Univ. Arizona Press, 2015).

A review article that presents spectral types of asteroids.

3. Watanabe, S. et al. Hayabusa2 arrives at the carbonaceous asteroid 162173 Ryugu-a spinning top-shaped rubble pile. Science $\mathbf{3 6 4}$, 268-272 (2019).

This paper reports initial data on the C-type asteroid Ryugu, collected by the Hayabusa2 spacecraft.

4. Brearley, A. J. \& Jones R. H. in Planetary Materials (ed, Papike, J. J.) Ch. 3 (Mineralogical Society of America, 1998).

A review article on chondritic meteorites.

5. Britt, D. T. \& Consolmagno, G. J. Stony meteorite porosities and densities: A review of the data through 2001. Meteorit. Planet. Sci. 38, 1161-1180 (2003).

A review article that presents the densities of meteorites.

\section{BEHIND THE PAPER}

It was more than six years after the samples were returned by Hayabusa2 that we started to prepare for their curation. Our facility was previously used to analyse samples that Hayabusa returned from Itokawa, but we added in some key functions. In designing new clean chambers for the facility, it was challenging to develop a sample extraction mechanism that enabled us to recover a few small particles of Ryugu from the container of the sample catcher under vacuum. We developed tools to unscrew and remove the bolts that fastened the cover plate of the catcher, and to remove the cover plate with an electrostatic chuck. We tested the upgraded facility, during the difficult time of the COVID-19 pandemic. Our 'eureka!' moment was when we successfully removed the cover plate of the catcher and found thousands of black Ryugu particles inside it. T.Y. and T.O.

\section{FROM THE EDITOR}

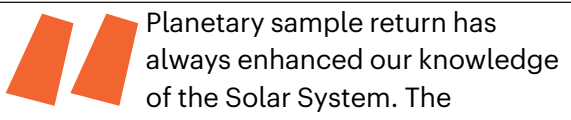

Hayabusa2 mission is no exception, as it allowed researchers to get a close look at a carbonaceous asteroid for the first time. This work presents the first preliminary results on the asteroid and gives clues on its small-scale properties and composition, which will be key to determining the details of its formation and evolution." Luca Maltagliati, Senior Editor, Nature Astronomy. 